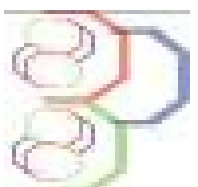

Journal of Applied Biosciences 86:7966- 7975

ISSN 1997-5902

\title{
Valorisation du lentisque «Pistacia lentiscus L.»: Étude ethnobotanique, Screening phytochimique et pouvoir antibactérien
}

\author{
Mohamed BAMMOU 1, Amine DAOUDI 1, Ikram SLIMANI', Mariam NAJEM'1, El Houssine \\ Bouiamrine ${ }^{1}$, Jamal IBIJBIJEN ${ }^{1}$ et Laila NASSIRI ${ }^{1}$ \\ 1 : Équipe de Microbiologie du Sol et de l'Environnement, Département de Biologie, Faculté des Sciences, \\ Université Moulay Ismail, BP 11201 Zitoune, Meknès. \\ Auteur correspondant : nassiri.laila@yahoo.fr et bammou.mohamed@gmail.com
}

Original submitted in on $11^{\text {th }}$ December 2014. Published online at www.m.elewa.org on 28 $8^{\text {th }}$ February 2015 http://dx.doi.org/10.4314/jab.v86i1.4

\section{RESUME}

Objectif : Cette étude consiste en une contribution à la valorisation du lentisque, très répandu dans la région de Meknès via une investigation ethnobotanique, un screening phytochimique et des tests antibactériens.

Méthodologie et résultats : Une étude ethnobotanique a été menée à l'aide de fiches questionnaires auprès de la majorité des herboristes de la ville de Meknès; ceci, afin de récolter le maximum d'informations sur les vertus et usages traditionnels de $P$. lentiscus $L$. L'enquête a révélé que le lentisque est couramment utilisé en thérapie $(96 \%)$, surtout dans le traitement des affections gastrointestinales $(88 \%)$, et dermatologiques $(28 \%)$. Le feuillage et les jeunes rameaux sont utilisés sous forme de tisane $(50 \%)$ et d'huiles essentielles (33\%). A travers l'investigation phytochimique et des tests d'activité antibactérienne conduits avec des extraits de feuilles et de jeunes rameaux, il apparait que $P$. lentiscus L. est riche en métabolites secondaires. De son côté, l'huile essentielle et les différents extraits testés sont dotés d'une activité antibactérienne considérable.

Conclusion et application de la recherche : L'étude ethnobotanique nous a permis de dévoiler le large éventail d'utilisation du feuillage de $P$. lentiscus $L$. dans le traitement de pathologies, notamment celles du tube digestif. Aussi, $P$. lentiscus $L$. est potentiellement important grâce à ses atouts en termes de biomasse, d'abondance sur le terrain, de richesse en métabolites secondaires (tanins, flavonoïdes, Stérols, triterpènes, et saponosides), d'effets antibactériens et de faible toxicité en rapport avec l'absence d'alcaloïdes et d'hétérosides cyanogénétiques.

Mots clés : Pistacia lentiscus L., Enquête ethnobotanique, Screening phytochimique, Activité antibactérienne, Meknès, Maroc

\begin{abstract}
Objective: The present study is a contribution to the popularization through an ethno botanical investigation, phytochemical screening and antibacterial tests of mastic tree "Pistacia lentiscus L." widespread in Meknes region.

Methods and results: A survey of most of Meknes town herbalists was conducted using a questionnaire in order to collect as much information on traditional virtues and uses of Pistacia lentiscus L. That investigation revealed that mastic tree is frequently used in therapy (96\%), especially to treat digestive diseases (88\%) and skin pains (28\%). Leaves and young twigs are the most used parts mainly under in
\end{abstract}


the form of an herb tea (50\%) and essential oils (33\%). The phytochemical screening and antibacterial tests undertaken on leaves and young twig showed that Pistacia lentiscus $L$. is rich in secondary metabolites. Also, essential oils and the different plant extracts tested had an important antibacterial activity.

Conclusion and application of research: Ethnobotany study revealed the wide range of uses of the foliage of $P$. lentiscus $L$. in the treatment of diseases, including those of the digestive tract. Also, $P$. lentiscus $L$. is potentially important because of its biomass, abundance in secondary metabolites (tannins, flavonoids, sterols, triterpenes and saponins) antibacterial effects and low toxicity related to the absence of alkaloids and cyanogenic glycosides.

Keywords: Ethnobotany, Pistacia lentiscus L., antibacterial activity, phytochemical screening, Meknes, Morocco.

\section{INTRODUCTION}

Le pistachier lentisque " Pistacia Lentiscus L. ", couramment appelé «Dro» en arabe local est un arbrisseau ramifié de trois mètres de hauteur, à odeur de résine fortement âcre (More et White, 2005). Ses feuilles composées paripennées et persistantes le distinguent des deux autres espèces spontanées au Maroc, $P$. atlantica Desf et $P$. terebinthus L. (Fennane et al., 2007). Autochtone au Maroc, on le rencontre en plaines, basses et moyennes montagnes, sous bioclimats semi-aride, subhumide, humide et perhumide (Fennane et al., 2007) ; les variantes thermiques sont chaude à fraiche et les substrats sont de type marneux ou argileux (Benabid, 2000). Du point de vue étage de végétation, le lentisque s'encarte en infra méditerranéen et thermo méditerranéen (Aafi et al., 2002; Benabid, 2000). Quant à sa biogéographie, il se rencontre en Atlas saharien, Anti-Atlas, Haut Atlas, Moyen Atlas, Rif, Maroc atlantique moyen, Maroc atlantique nord, monts de l'oriental et littoral de la méditerranée (Fennane et al., 2007). Aussi, avec la filaire, il forme l'association phillereo latifoliae-Pistacietum lentisci, correspondant à un matorral issu de la dégradation de forêts thermo méditerranéennes subhumides (Benabid, 2000). Par ailleurs, Pistacia lentiscus L. est connu pour ses propriétés médicinales

\section{MATÉRIEL ET MÉTHODES}

Étude ethnobotanique : L'enquête ethnobotanique concernant $P$. lentiscus $L$. a été menée auprès de 25 herboristes répartis dans les différents quartiers de la ville de Meknès via un questionnaire préétabli et un entretien direct, qui a duré en moyenne une heure par personne. Le choix des personnes enquêtées a été basé sur leur niveau intellectuel et leur expérience dans le domaine des plantes médicinales. Aussi, depuis l'antiquité; en effet, les médecines traditionnelles pratiquées de part et d'autre des rives de la méditerranée, attribuent au lentisque des vertus dans le traitement des ulcères, l'hypertension, la toux, les maux de gorge, l'eczéma, des calculs rénaux et la jaunisse (Gardeli et al., 2008). Le mastic de Pistacia a été utilisé par les guérisseurs traditionnels pour le soulagement des douleurs abdominales, des maux d'estomac, la dyspepsie et l'ulcère gastroduodénal (Al-Habbal et al., 1984). Plusieurs études ont également signalé que l'huile essentielle des parties aériennes de Pistacia lentiscus $L$. possède des propriétés antifongiques et antibactériennes appréciables (Gardeli et al., 2008 ; Kordali et al., 2003 ; Bonsignore et al., 1998). L'huile de fruits du lentisque est souvent utilisée comme un remède d'application externe locale sous forme d'onguent pour soigner les brûlures ou les douleurs dorsales (Bellakhdar, 1997). Aussi, l'objectif de ce travail consiste en une contribution à la valorisation du lentisque, très répandu dans la région de Meknès via une investigation ethnobotanique, un screening phytochimique et des tests antibactériens.

toutes les informations concernant la plante objet de l'étude ont été recueillies et statistiquement analysées par le tableur Excel.

Récolte et identification de la plante : La plante a été récoltée dans les environs de Meknès et soigneusement identifiée par l'équipe de botanique de la faculté des sciences de Meknès. 


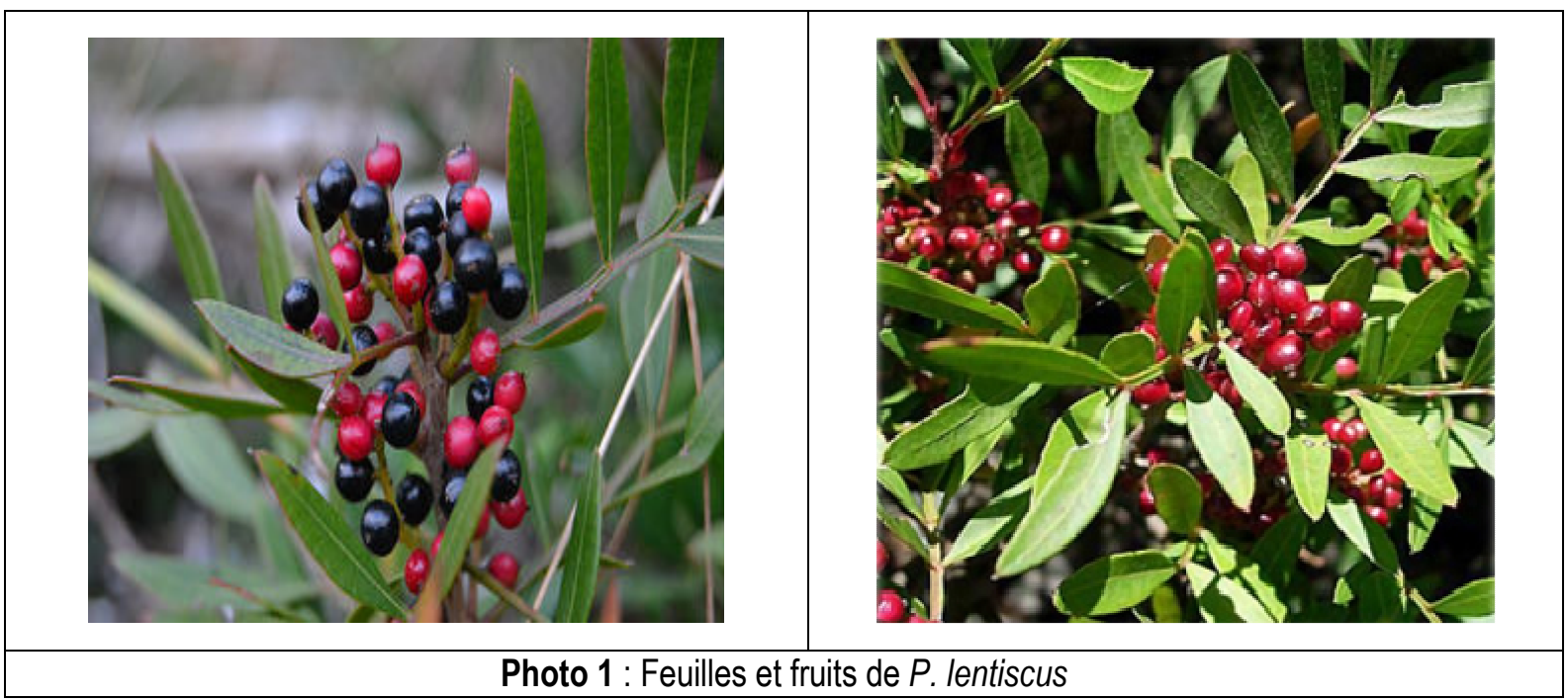

Des feuilles et de jeunes rameaux ont été fraîchement récoltés au mois de Mai 2014 sur des pieds spontanés de lentisque. Après un séchage à l'ombre en endroit sec et aéré, les échantillons ont été récupérés dans des sacs propres et stockés à l'abri de la lumière et de l'humidité

Extraction des huiles essentielles : L'extraction des huiles essentielles a été effectuée par hydrodistillation dans un appareil de type Clevenger (Clevenger, 1928). Trois distillations ont été réalisées par ébullition dans un ballon de $100 \mathrm{~g}$ de matériel végétal frais avec un litre d'eau pendant quatre heures.

Extraction par soxlhet : L'extraction des substances naturelles à partir de la plante a été effectuée en utilisant un appareil Soxlhet; quatre solvants ont été utilisés dans l'ordre croissant de polarité : Ether de pétrole (EP), Acétate d'éthyle (AE), Méthanol (Mét) et Eau $(\mathrm{Aq})$; les quantités de matériel végétal placées dans les cartouches furent d'environ $50 \mathrm{~g}$ pour $150 \mathrm{ml}$ de solvant (Dohou et al., 2003).

Infusion et décoction : L'infusé et le décocté ont été préparés selon la méthode adoptée par Sqalli et al. en utilisant $10 \mathrm{~g}$ de poudre par $100 \mathrm{ml}$ d'eau (Sqalli et al., 2007).

Screening phytochimique : II s'agit d'une étude qualitative visant la recherche des principaux groupes chimiques (Alcaloïdes, polyphénols, flavonoïdes, tanins, saponosides, composés réducteurs, composés cyanogénétiques...). Les tests de caractérisation sont basés sur des réactions de précipitation et de complexassion avec formation de complexes insolubles et colorés. La coloration observée est provoquée par l'utilisation d'un réactif approprié et est due généralement à la formation d'une conjugaison ou d'une instauration dans une molécule. Nous nous sommes servis des techniques analytiques décrites dans les travaux de (Bekro et al., 2007), (Dohou et al., 2003), (Longanga Otshudi et al., 2000), (Tona et al., 1998) et (Randerath et Nguyen, 1971).

Ainsi, les alcaloïdes ont été caractérisés grâce aux réactifs de Mayer et de Dragendorff; un extrait sulfurique a été préparé à partir de $5 \mathrm{~g}$ de poudre et de $25 \mathrm{ml}$ de $\mathrm{H}_{2} \mathrm{SO}_{4}$ à $10 \%$. Après agitation, il fut laissé en macération pendant $24 \mathrm{~h}$ à la température ambiante du laboratoire, puis filtré sur papier filtre et lavé à l'eau distillée de manière à obtenir $25 \mathrm{ml}$. Le procédé a consisté à utiliser $1 \mathrm{ml}$ de ce filtrat dans 2 tubes à essai et à ajouter 5 gouttes du réactif de Dragendorff dans le premier tube et 5 gouttes du réactif de Mayer dans le deuxième tube, l'apparition de précipités indique la présence d'alcaloïdes.

La recherche des tanins catéchiques a été réalisée via recours au réactif de Stiasny; Cinq (5) $\mathrm{ml}$ de chaque extrait ont été évaporés à sec. Après ajout de $15 \mathrm{ml}$ du réactif de Stiasny au résidu, le mélange fut maintenu au bain-marie à $80^{\circ} \mathrm{C}$ pendant $30 \mathrm{~min}$. L'observation d'un précipité en gros flocons caractérise les tanins catéchiques. Quant aux tanins galliques, la solution précédente a été filtrée et le filtrat recueilli et saturé d'acétate de sodium. L'addition de 3 gouttes de $\mathrm{FeCl}_{3}$ provoquerait l'apparition d'une coloration bleu-noir intense, signe de la présence de tanins galliques. Les flavonoïdes ont été recherchés par la réaction à la Cyanidine ; à $5 \mathrm{ml}$ de chaque extrait de l'infusé (à $5 \%$ ) présentant une coloration plus ou moins foncée furent ajoutés $5 \mathrm{ml}$ d'acide sulfurique à $10 \%$ puis une base $\left(\mathrm{NH}_{4} \mathrm{OH}\right)$. $\mathrm{Si}$ la coloration augmente par acidification, puis vire au bleu-violacé en milieu basique, la présence d'anthocyanes est confirmée. Dans un tube à essai, 5 $\mathrm{ml}$ d'alcool chlorhydrique, $1 \mathrm{ml}$ d'alcool iso-amylique 
et quelques copeaux de Magnésium ont été ajoutés à $5 \mathrm{ml}$ de chaque extrait de l'infusé ; l'apparition d'une coloration rose orangée, rose violacée ou rouge indique respectivement la présence de flavones, flavanones ou flavanols et flavanonols. La même réaction fut réalisée, mais cette sans copeaux de Magnésium; le mélange a été porté à ébullition au bain-marie pendant $15 \mathrm{mn}$. L'apparition de coloration rouge cerise ou violacée permet de déduire la présence de Leucoanthocyanes. La formation d'une teinte brun-rouge indique la présence de catéchols.

Pour la mise en évidence des composés réducteurs, $5 \mathrm{ml}$ de décocté aqueux à $10 \%$ ont été introduits dans un bécher de $100 \mathrm{ml}$ et évaporés à sec au bainmarie. Au résidu, fut ajouté $1 \mathrm{ml}$ du réactif de Fehling et l'obtention d'un précipité rouge-brique indique la présence de composés réducteurs. Les oses et holosides ont été recherchés par introduction de $5 \mathrm{ml}$ du décocté à $10 \%$ dans un bécher de $100 \mathrm{ml}$ et évaporation à sec dans un bain-marie. 2 à 3 gouttes de $\mathrm{H}_{2} \mathrm{SO}_{4}$ concentré furent ajoutées au résidu; Après $5 \mathrm{mn}, 3$ à 4 gouttes d'Éthanol saturé avec du Thymol furent ajoutées. Le développement d'une coloration rouge révèle la présence d'oses et holosides. Les mucilages ont été également caractérisés par l'introduction de $1 \mathrm{ml}$ du décocté à $10 \%$ dans un tube à essai et ajout de $5 \mathrm{ml}$ d'Éthanol absolu. Après une dizaine de minutes, l'obtention d'un précipité floconneux par mélange, indique la présence de mucilages. Pour la mise en évidence des composés cyanogénétiques, trois grammes de matériel végétal frais ont été mouillés avec quelques gouttes de Chloroforme $\left(\mathrm{CHCl}_{3}\right)$ dans un tube à essai où fut insérée une bandelette de papier filtre imprégnée de Picrate de Sodium. Le tube fut alors placé dans un bain-marie à $35^{\circ} \mathrm{C}$ pendant 3 heures. Un virage au rouge de la bandelette indique la présence des composés cyanogénétiques.

De son côté, la détection des Stérols et des Triterpènes a été réalisée sur l'extrait obtenu à partir de $1 \mathrm{~g}$ de poudre et $20 \mathrm{ml}$ d'Ether laissés en macération pendant 24 heures, puis filtrés et complétés à $20 \mathrm{ml}$ avec de l'éther. Après avoir évaporé à sec $10 \mathrm{ml}$ de l'extrait, le résidu a été dissout dans $1 \mathrm{ml}$ d'Anhydride Acétique, puis $1 \mathrm{ml}$ de Chloroforme et recueilli dans deux tubes à essai, dont l'un sert de référence. A l'aide d'une pipette, 1 à $2 \mathrm{ml}$ de $\mathrm{H}_{2} \mathrm{SO}_{4}$ concentré ont été déposés au fond du tube

\section{RESULTATS ET DISCUSSION \\ Étude ethnobotanique}

Partie de la plante utilisée et formes d'emploi : D'après les résultats de l'enquête ethnobotanique sur le lentisque, il apparait que tous les herboristes à essai sans agitation. A la zone de contact des deux liquides, il y a formation d'un anneau rouge brunâtre ou violet; la couche surnageant devenant verte ou violette révèle la présence de stérols et de triterpènes. En fin, pour la recherche des saponosides, $10 \mathrm{ml}$ de l'extrait total aqueux ont été versés dans un tube à essai ; le tube, agité pendant $15 \mathrm{~s}$ a été laissé au repos durant $15 \mathrm{~min}$. Une hauteur de mousse persistante, supérieure à $1 \mathrm{~cm}$ indique la présence de saponosides.

Matériel bactérien : Le matériel microbien utilisé est composé de quatre souches pathogènes isolées cliniquement à l'hôpital Mohamed V de Meknès et dont la sensibilité aux antibiotiques est déterminée par le test d'antibiogramme selon les recommandations du comité de l'antibiogramme de la Société Française de Microbiologie (Bonnet et al., 2010). II s'agit du cocci Gram (+) Staphylococcus aureus et de bacilles et de coccobacilles Gram (-), Escherichia coli, Klebsiella pneumoniae, Proteus mirabilis.

Évaluation de l'activité antibactérienne des extraits du lentisque

Préparation de l'inoculum: Des cultures bactériennes sur gélose non inhibitrice, âgées de 24 heures ont été mises en suspension dans de l'eau physiologique stérile $(0.9 \%)$. La turbidité de l'inoculum fut ajustée à $0.5 \mathrm{Mc}$ Farland, ce qui correspond à un inoculum de $10^{8} \mathrm{CFU} / \mathrm{ml}$.

Technique de diffusion en milieu gélosé : Ce test a été réalisé par la méthode de diffusion en milieu solide qui consiste en la détermination des diamètres des zones d'inhibition (Gupta et al., 2010).

Des disques de $6 \mathrm{~mm}$ de diamètre découpés sur papier Wattman $\mathrm{N}^{\circ} 1$, stérilisés et imprégnés à raison de $25 \mu \mathrm{L}$ d'extraits à tester par disque ont été déposés à la surface d'un milieu préalablement ensemencé. Après incubation à $37^{\circ} \mathrm{C}$ pendant $24 \mathrm{~h}$, les zones d'inhibition formées autour des disques ont été mesurées à l'aide d'un pied à coulisse. Chaque essai a été répété trois fois dans les mêmes conditions d'expérimentation.

Préparation des extraits à tester : A partir des extraits réalisés, des solutions mères de $10 \%$ ont été préparées. Les extraits organiques furent dissous dans le DMSO (2\%) et l'extrait aqueux, le décocté et l'infusé dans l'eau distillée stérile.

enquêtés recommandent et vendent la feuille $(100 \%)$ et seuls $4 \%$ d'entre- eux ont en plus recours à la tige [Fig.2] . La fréquence élevée d'utilisation des feuilles peut être expliquée par le fait qu'elles sont le 
siège de la photosynthèse et parfois du stockage des métabolites secondaires responsables des propriétés biologiques de la plante (Bigendako-Polygenis and Lejoly, 1990) en plus de l'aisance et la rapidité de leur récolte (Bitsindou and Bouquet, 1996). Quant à la forme d'emploi [Fig.3], la plus fréquente est la tisane

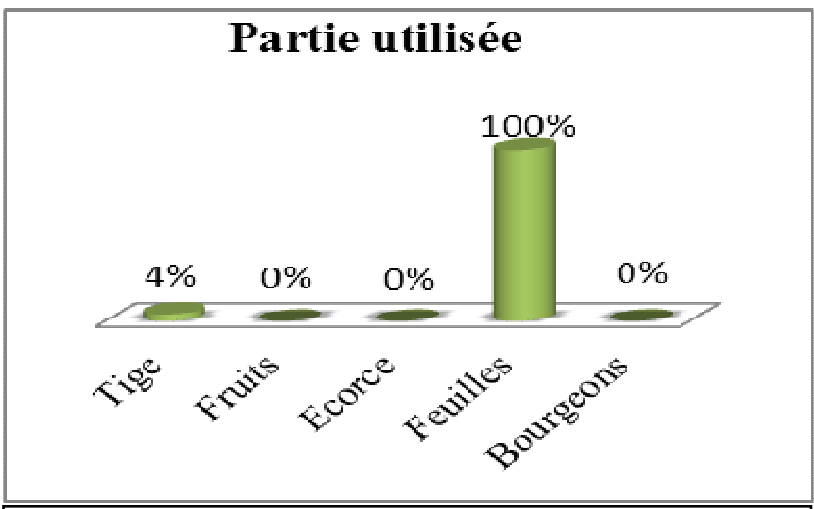

Figure 2 : Répartition selon la partie utilisée

Répartition selon la dose utilisée et le nombre de prises par jour : En ce qui concerne la dose, la plus prescrite est la poignée $(76 \%)$ suivie de la cuillerée (20\%) [Fig.4]. la posologie n'est donc pas exacte
$(50 \%)$; selon les herboristes enquêtés, celle-ci permet de faciliter le mode d'administration de la plante et d'avoir des résultats rapides et efficaces. Le lentisque est également utilisé sous forme d'huile essentielle $(33 \%)$ et de poudre $(7 \%)$.

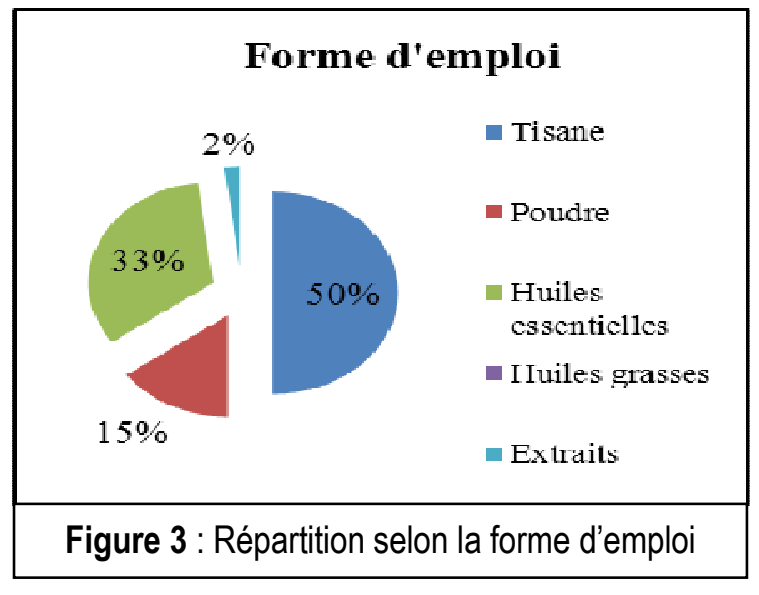

chez les herboristes d'où le risque d'effets néfastes inhérents au surdosage (Benkhnigue et al., 2010) ou encore d'inefficacité en rapport avec une dose insuffisante.
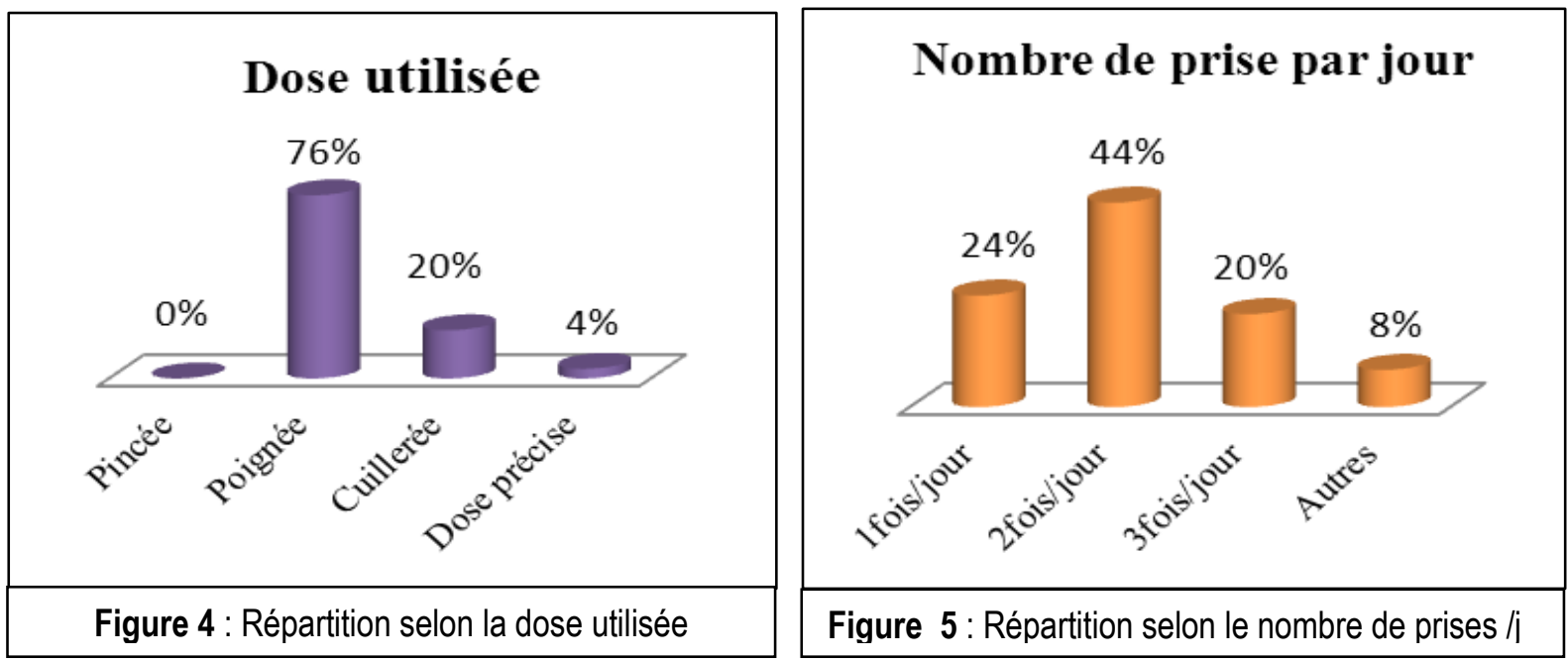

Par ailleurs, la prise la plus conseillée est deux fois par jour (44\%) sinon une fois $(24 \%)$ à trois fois par jour (20\%) [Fig.5].

Répartition selon les pathologies traitées et la toxicité : L'enquête menée auprès d'herboristes nous a permis de répertorier un certain nombre de maladies traitées par Pistacia lentiscus L. [Fig.6] ; il s'agit essentiellement d'affections du tube digestif (88\%). Aussi, $28 \%$ des enquêtés ont recours aux feuilles du lentisque pour le traitement d'affections dermatologiques, $20 \%$ pour les affections génitourinaires, et $16 \%$ aussi bien pour les pathologies bucco-dentaires que métaboliques. Par ailleurs, selon $8 \%$ seulement des enquêtés, la plante serait toxique [Fig.7]. 


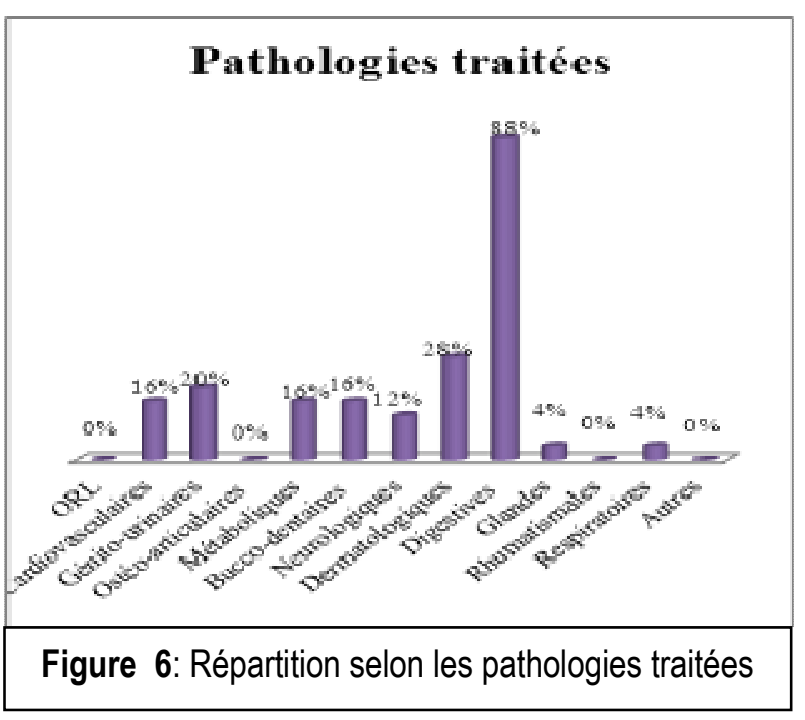

\section{Étude phytochimique}

Teneur en eau et rendement en huiles essentielles : La teneur en eau des feuilles et des jeunes rameaux de Pistacia lentiscus $L$. a été réalisée en triplicata ; elle est de l'ordre de $63.33 \pm 1.15 \%$. Le rendement en huiles essentielles des feuilles et des petits rameaux de Pistacia lentiscus $L$. est faible ; il est de l'ordre de $0.16 \%$. Ceci corrobore les résultats trouvés par Dob et al. (2006) qui étaient de l'ordre de 0,11 à $0,20 \%$ et celui de $0,14 \%$ obtenu par Amhamdi et al. (2009).

Screening phytochimique : Le screening phytochimique des feuilles et des petits rameaux du lentisque a mis en évidence la présence de plusieurs composés chimiques réputées avoir des activités biologiques intéressantes [Tableau 1]. II s'agit des

\section{Toxicité}

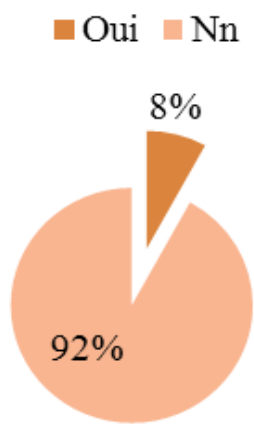

Figure 7 : Répartition selon la toxicité

substances polyphénoliques dont les tanins chatéchiques et galliques, des flavonoïdes (anthocyanes, flavones et leucoanthocyanes), des stérols et triterpènes, des saponosides et en fin les composés réducteurs (oses, holosides et mucilage). L'absence totale des hétérosides cyanogénétiques diminue fortement les risques toxicologiques liés à l'usage de Pistacia lentiscus L. (Andersen and Markham, 2010), ce qui est en accord avec les résultats de l'enquête ethnobotanique rapportant que peu d'herboristes sont en faveur d'une toxicité du lentisque.

De son côté, la présence des flavonoïdes confirme l'effet hépatoprotecteur, antispasmodique, antimicrobien et veinoactif alloué aux feuilles de Pistacia lentiscus L. (Andersen and Markham, 2010).

Tableau 1 : Résultats du screening phytochimique

\begin{tabular}{|c|c|c|}
\hline & Métabolites secondaires & Réaction \\
\hline Alcaloïdes & & - \\
\hline Tanine & Chatéchiques & ++++ \\
\hline S Iamits & Galliques & +++ \\
\hline & Anthocyanes & ++ \\
\hline & Flavones & ++ \\
\hline & Flavanones & - \\
\hline Flavonoldes & Flavanonols & - \\
\hline & Leucoanthocyanes & +++ \\
\hline & Catéchols & - \\
\hline & Oses et holosides & +++ \\
\hline Composés réducteurs & Mucilages & ++ \\
\hline & Hétérosides cyanogénétiques & - \\
\hline Ternénoïdes & Stérols et triterpènes & ++ \\
\hline Terpenoides & Saponosides & +++ \\
\hline
\end{tabular}

++++: Réaction très positive; +++: Réaction positive; ++ Réaction moyennement positive;+: Réaction douteuse ; - : Test négatif 
Les tanins galliques sont tenus comme bons remèdes dans le traitement des maladies respiratoires et contre la toux; par voix interne, les tanins exercent une activité antidiarrhéique certaine (Romani et al., 2002). Ces propriétés antiseptiques, antibactériennes et antifongiques sont clairement démontrées dans le traitement des diarrhées infectieuses et des dermatites ce qui est en corrélation avec l'usage traditionnel; en effet, $88 \%$ des herboristes touchés par notre enquête recommandent l'utilisation de Pistacia lentiscus L. pour le traitement des affections du tube digestif et $28 \%$ d'entre eux pour traiter les affections dermatologiques. Ces résultats corroborent ceux mentionnés par d'autres chercheurs, notamment Romani et al. (2002).

\section{Activité antibactérienne}

Effets des extraits et de l'huile essentielle : II apparait que l'HE de Pistacia lentiscus L. n'a aucun effet sur Escherichia coli, Klebsiella pneumoniae et Proteus mirabilis; seul Staphylococcus aureus laisse voir une légère sensibilité (diamètre d'inhibition: $10 \pm 1.00 \mathrm{~mm}$ ) qui reste en fait inferieure à l'effet de la Gentamicine (CN) [Tableau 2 ; Fig. 8]. Aussi, ni le décocté ni l'infusé des feuilles et des jeunes rameaux n'ont eu d'effet sur Escherichia coli et Klebsiella pneumoniae [Tableau 2]. Par contre, Staphylococcus aureus et Proteus mirabilis laissent voir une certaine sensibilité ; l'effet le plus important étant obtenu avec le décocté sur Staphylococcus aureus « $28 \pm 1.00$ $\mathrm{mm} »$, dépassant de loin l'effet de la Gentamicine « $19 \mathrm{~mm}$ » [Tableau 2 ; Fig. 8, 9,10]. L'utilisation de solvants à polarité différente permet de séparer des composés selon leur degré de solubilité dans le solvant d'extraction. Ainsi, la fraction aqueuse, méthanolique et celui d'acétate d'éthyle se sont révélés actifs avec un degré différent, lié probablement au contenu des extraits en substances à activité antimicrobienne [fig. 11]. L'extrait éthérique semble le plus inactif sur toutes les souches testées ce qui peut être expliqué par l'absence d'activité de ses composés apolaires qui sont essentiellement des matières grasses. II apparaît aussi que Staphylococcus aureus (gram positive) est la bactérie la plus susceptible par comparaison avec les autres souches (gram négative) ; ceci peut être attribué à la différence de la structure entre les bactéries gram positives et les bactéries gram négatives. La paroi cellulaire des bactéries gram positives est constituée par une seule couche alors que celle des grams négatifs a une structure multicouche liée par une membrane cellulaire externe (Ali-Shtayeh et al., 1998). Aussi, contrairement aux extraits fractionnés et à l'huile essentielle, les extraits bruts sont les seuls à avoir montré un effet inhibiteur sur Proteus mirabilis. Cet effet est très pertinent notamment pour l'infusé «27.00 $\pm 1.00 \mathrm{~mm} » \mathrm{car}$ il dépasse l'effet de l'Amoxicilline (AMC) « $24 \mathrm{~mm} »$.

Tableau 2 : Diamètres des zones d'inhibition en mm obtenus avec l'huile essentielle et les extraits des feuilles et jeunes rameaux de Pistacia lentiscus L.

\begin{tabular}{|c|c|c|c|c|c|}
\hline \multirow{2}{*}{\multicolumn{2}{|c|}{ 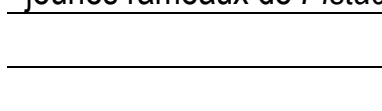 }} & \multicolumn{4}{|c|}{ Souches bactériennes } \\
\hline & & S.aureus & E.coli & K. pneumoniae & P. mirabilis \\
\hline \multicolumn{2}{|l|}{ Décocté } & $28.00 \pm 1.00$ & NA & NA & $22.33 \pm 0.57$ \\
\hline \multicolumn{2}{|l|}{ Infusé } & $27.00 \pm 1.00$ & NA & NA & $27.00 \pm 1.00$ \\
\hline \multicolumn{2}{|c|}{ Huile essentielle } & $10.00 \pm 1.00$ & NA & NA & NA \\
\hline \multicolumn{2}{|c|}{ Fraction Aqueuse } & $8.66 \pm 0.57$ & NA & NA & NA \\
\hline \multicolumn{2}{|c|}{ E. Méthanolique } & $8.66 \pm 1.53$ & NA & NA & NA \\
\hline \multicolumn{2}{|c|}{ E. Acétate d'éthyle } & $8.00 \pm 0.00$ & NA & NA & NA \\
\hline \multicolumn{2}{|c|}{ E. Ether de pétrole } & NA & NA & NA & NA \\
\hline \multirow{4}{*}{ Antibiotique } & $\mathrm{OX}_{5}$ & $0 \pm 0.00$ & NT & NT & NT \\
\hline & $\mathrm{CN}_{10}$ & $19 \pm 0.00$ & NT & NT & NT \\
\hline & $\mathrm{IPM}_{5}$ & NT & $24 \pm 1.00$ & 24 & $24 \pm 1.00$ \\
\hline & $\mathrm{AMC}_{30}$ & NT & NT & NT & $0 \pm 0.00$ \\
\hline
\end{tabular}

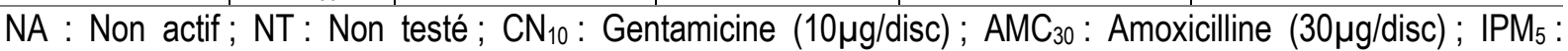
Imipénème $(5 \mu \mathrm{g} / \mathrm{disc}) ; 0 X_{5}$ : Oxacilline $(5 \mu \mathrm{g} / \mathrm{disc})$. 


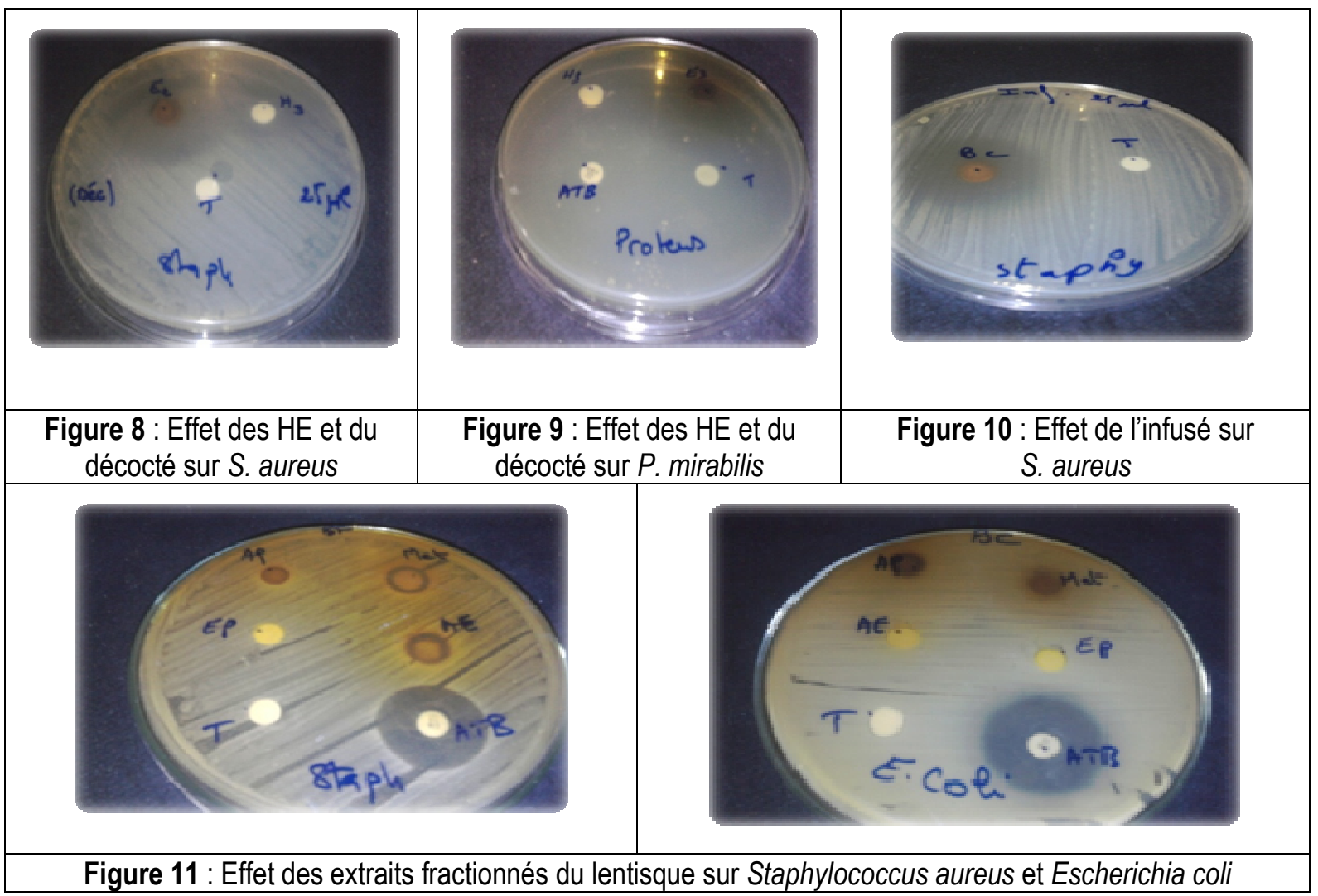

En comparant l'huile essentielle, extraits totaux et extraits fractionnés, on peut dire que les extraits totaux sont les plus puissants; donc une plante entière est plus efficace que la somme de ses composants; en effet, les plantes contiennent des centaines voire des milliers de substances chimiques actives. D'ailleurs la phytothérapie recommande d'utiliser la plante entière, appelée aussi "Totum" plutôt que des extraits obtenus en laboratoire (Iserin et al., 2001). De plus, l'efficacité du décocté ou de l'infusé diffère selon le type de bactérie ; ainsi, pour

\section{CONCLUSION}

L'étude ethnobotanique menée auprès des herboristes de la ville de Meknès sur $P$. lentiscus $L$. nous a permis de dévoiler le large éventail de l'utilisation de son feuillage dans le traitement de pathologies, notamment celles du tube digestif. Aussi, Pistacia lentiscus $L$. est potentiellement importante grâce à ses atouts en termes de biomasse,

\section{BIBLIOGRAPHIE}

Aafi A., Taleb MS., Fechtal M, 2002. Espèces Remarquables de la Flore du Maroc, Rabat: Édition MCEF : $146 \mathrm{p}$.

Ali-Shtayeh MS., Yaghmour RMR., Faidi YR., Salem K., Al-Nuri MA, 1998. Antimicrobial Activity of 20 Plants Used in Folkloric Medicine in the Palestinian Area. Journal of
Staphylococcus aureus, le décocté des feuilles est le plus puissant alors que pour Proteus mirabilis c'est plutôt l'infusé. En outre, il est à noter que l'effet des extraits et des huiles essentielles est puissant par rapport à l'Oxacilline (OX 5) et l'Amoxicilline (AMC) qui semblent inactifs sur respectivement Staphylococcus aureus et Proteus mirabilis. Enfin, l'absence de l'effet à la fois des extraits et aussi de l'huile sur Klebsiella pneumoniae et Escherichia coli [fig.11] peut être expliqué par la multi-résistance de ces souches.

d'abondance sur le terrain, de richesse en métabolites secondaires, essentiellement les polyphénols et les flavonoïdes, d'effets antibactériens et de faible toxicité en rapport avec l'absence d'alcaloïdes et d'hétérosides cyanogénétiques ce qui atténuerait l'effet de l'augmentation de la dose.

Ethnopharmacology : Vol. 60, №. 3, 265271.

Al-Habbal, Jamil M., Al-Habbal Z., Umer Huwez F, 1984. A Double-Blind Controlled Clinical Trial of Mastic and Placebo in the Treatment of Duodenal Ulcer. Clinical and experimental 
pharmacology and physiology : Vol. 11, No. $5,541-544$.

Amhamdi H., Aouinti F., Wathelet JP., Elbachiri A, 2009. Chemical Composition of the Essential Oil of Pistacia Lentiscus L. From Eastern Morocco, Rec. Nat. Prod : Vol. 3, No. 2, 9095.

Andersen OM. and Markham KR, 2010. Flavonoids: Chemistry, Biochemistry and Applications, CRC Press : 472-551.

Bekro YA., Mamyrbekova JA., Boua BB., Ehile EE, 2007. Étude Ethnobotanique Et Screening Phytochimique de Caesalpinia Benthamiana (Baill.) Herend. rt Zarucchi (Caesalpiniaceae). Sciences \& Nature : Vol. 4, No. 2, 217-225.

Bellakhdar J, 1997. La Pharmacopee Marocaine Traditionnelle: Medecine Arabe Et Savoirs Populaires. Editions Le Fennec, (Ed.) (Eds.), Ibis Press, Casablanca, Morocco : 764p.

Benabid A, 1982. Étude Phytoècologique, Biogéographique et Dynamique des Associations et Séries Sylvatiques du Rif Occidental (Maroc). Fac. Sc. Tech. St. Jérome. Thesis Univ. D'Aix-Marseille.

Benabid A, 2000. Flore Et Écosystèmes Du Maroc: Évaluation Et Préservation De La Biodiversité, Paris: Editions Ibis Press : 359 $p$.

Benkhnigue O., Zidane L., Fadli M., Elyacoubi H., Rochdi A., Douira A, 2010. Étude Ethnobotanique des Plantes Médicinales dans la Région de Mechraâ Bel Ksiri (Région Du Gharb Du Maroc), Acta Botanica Barcinonensia : Vol. 53, 191-216.

Bigendako-Polygenis MJ. and Lejoly J, 1990. La Pharmacopée Traditionelle Au Burundi. Pesticides et médicaments en santé animale : 425-442.

Bitsindou M. and Bouquet A, 2004. Enquêtes sur la Phytothérapie Traditionnelle à Kindamba et Odzala, Betti, JL. An ethnobotanical study of medicinal plants among the Baka pygmies in the Dja Biosphere Reserve, Cameroon. African Study Monographs : Vol. 25, No. 1, 1-27, 1996.

Bonnet R., Cavallo JD., Chardon H., Chidiac C., Courvalin $\mathrm{P}_{\text {., }}$ Dabernat $\mathrm{H}_{\text {., }}$ Drugeon $\mathrm{H}_{\text {., }}$ Dubreuil L., Guery B, 2010. Comité de l'antibiogramme de la Société Française de Microbiologie, (Ed.), Recommandations.

Bonsignore L., Cottiglia F., Loy G, 1998. Antibacterial Activity of Pistacia Lentiscus Aerial Parts. Fitoterapia : Vol. 69, No. 6, 537-538.
Clevenger JF, 1928. Apparatus for the Determination of Volatile Oil. Journal of the American Pharmaceutical Association : Vol. 17, No. 4, 345-349.

Dob T., Dahmane D., Chelghoum C, 2006. Chemical Composition of the Essential Oils of Pistacia Lentiscus L. From Algeria. Journal of Essential Oil Resear : Vol. 18, No. 3, 335338.

Dohou R., Yamni K., Tahrouch S., Hassani IL., Badoc A., Gmira N, 2003. Screening Phytochimique d'une Endemique Iberomarocaine, Thymelaea Lythroides. Bulletin-Societe de Pharmacie de Bordeaux: Vol. 142, No. 1/4, 61-78.

Fennane M., Ibn Tattou M., Ouyahya A., El Oualidi J, 2007. Flore Pratique du Maroc: Manuel de Détermination des Plantes Vasculaires. Travaux de I'Institut Scientifique, Rabat, série botanique : Vol. 2, No. 38. p 250.

Gardeli C., Vassiliki P., Athanasios M., Kibouris M., Komaitis M, 2008. Essential Oil Composition of Pistacia Lentiscus L. And Myrtus Communis L.: Évaluation of Antioxidant Capacity of Methanolic Extracts. Food chemistry : Vol. 107, No. 3, 1120-1130.

Gupta VK., Roy A., Nigam V.K., Mukherjee K, 2010. Antimicrobial Activity of Spondias Pinnata Resin. J. Med. Plants Res: Vol. 4, 16561661.

Iserin P., Masson M., Restellini JP., Ybert E., De Laage de Meux A., Moulard F., Zha Z., De la Roque R., De la Roque O., Vican P, 2001. Larousse des Plantes Médicinales: Identification, Préparation, Soins. Larousse : 10-12.

Kordali S., Cakir A., Zengin H., Duru ME, 2003. Antifungal Activities of the Leaves of Three Pistacia Species Grown in Turkey. Fitoterapia : Vol. 74, No. 1, 164-167.

Longanga Otshudi A., Foriers A., Vercruysse A., Van Zeebroeck A., Lauwers S, 2000. In Vitro Antimicrobial Activity of Six Medicinal Plants Traditionally Used for the Treatment of Dysentery and Diarrhoea in Democratic Republic of Congo (Drc). Phytomedicine : Vol. 7, No. 2, 167-172.

More D. and White J, 2005. Encyclopédie des Arbres: Plus de 1800 Espèces et Variétés du Monde, (Ed.), Flammarion : 18-24.

Kurt R. and Nguyen DT, 1971. Chromatographie Sur Couches Minces. Gauthier-villars.

Romani A., Pinelli P., Galardi C., Mulinacci N., Tattini M, 2002. Identification and Quantification of 
Galloyl Derivatives, Flavonoid Glycosides and Anthocyanins in Leaves of Pistacia Lentiscus L, Phytochemical Analysis : Vol. 13, No. 2, 79-86.

Sqalli H., El Ouarti A., Ennabili A., Ibnsouda S., Farah A., Haggoud A., Houari A., Iraqui M, 2007. Évaluation de l'effet Antimycobactérien de
Plantes du Centre-Nord du Maroc. Bull Soc Pharm : Vol. 146, 271-288.

Tona L., Kambu K., Ngimbi N., Cimanga K., Vlietinck AJ, 1998. Antiamoebic and Phytochemical Screening of Some Congolese Medicinal Plants. Journal of Ethnopharmacology : Vol. 61 , No. 1, 57-65. 